\title{
Risk Factors for Clinically Relevant Loosening of Percutaneous Pedicle Screws
}

\author{
Tetsuro Ohba ${ }^{1)}$, Shigeto Ebata ${ }^{1)}$, Hiroki Oba ${ }^{122}$, Kensuke Koyama ${ }^{1)}$ and Hirotaka Haro ${ }^{1)}$ \\ 1) Department of Orthopaedic Surgery, University of Yamanashi, Chuou-city, Japan \\ 2) Department of Orthopaedic Surgery, Shinshu University, School of Medicine, Matsumoto-city, Japan
}

\begin{abstract}
:
Introduction: (1) To evaluate the influence of pedicle screw loosening on clinical outcomes; (2) to clarify the association between the pull-out length and screw loosening 1 year after surgery; and (3) to determine radiographically which screw parameters predominantly influence the pull-out resistance of screws.

Methods: We analyzed 32 consecutive patients who underwent minimally invasive lumbar or thoracic spinal stabilization by intraoperative three-dimensional computed tomography (CT)-guided navigation without anterior reconstruction and were followed up for 1 year. The screw pull-out length was measured on axial CT images obtained both immediately after screw insertion and postoperatively. Loosening of screws and clinical outcomes were evaluated radiographically, clinically, and by CT 1 year after surgery.

Results: There were no significant differences in the mean age, sex, bone mineral density, mean stabilized length, and smoking habits of patients with (+) or without (-) loosening. The Oswestry Disability Index and the lumbar visual analog scale 1 year after surgery were significantly higher in patients with loosening $(+)$ than in those without $(-)$. The overall pedicle screw pull-out rate was $16.2 \%$ (47/290) of screws and the overall screw loosening rate was 15.2\% (44/290) of screws. Screws with loosening (+) had significantly lower (axial) trajectory angles and higher screw pull-out lengths than those without (-). Approximately $82 \%$ of loosened screws had been pulled out during rod connection.

Conclusions: A lower axial trajectory and an increased screw pull-out length after rod reduction are crucial risk factors for screw loosening.

Keywords:

screw loosening, percutaneous pedicle screw, intraoperative CT navigation, screw pull-out, screw trajectory angle

Spine Surg Relat Res 2019; 3(1): 79-85

dx.doi.org/10.22603/ssrr.2018-0018
\end{abstract}

\section{Introduction}

Posterior pedicle screw instrumentation has become the most frequently used surgical technique to treat spinal disorders ${ }^{1)}$. Recently, a minimally invasive spine surgery technique, percutaneous pedicle screw (PPS) placement, has been growing in popularity with the concept of an internal splint to achieve stability of the thoracolumbar spine in a variety of indications, such as vertebral fractures, vertebral osteomyelitis, and metastatic spinal tumors ${ }^{2}$. However, poor screw-to-bone fixation or local high strains at the bonescrew interface might cause clinical problems such as screw loosening ${ }^{3)}$. Numerous biomechanical studies of pedicle screw pull-out strength have been conducted using human cadaveric spine specimens, animal spine models, or bone models ${ }^{4-9)}$. However, these studies have remained limited because it is difficult to accommodate the wide variety of actual clinical factors.

High accuracy and a low rate of facet joint violation in patients undergoing PPS with intraoperative computed tomography (CT) (O-arm)-guided navigation (CT-IGN) have recently been demonstrated ${ }^{10)}$. In these cases, the positions of pedicle screws have been determined by CT both immediately after pedicle screw insertion and 1-7 days postoperatively. Therefore, the pull-out length of the pedicle screw during rod connection can be measured as the difference between the two CT images.

Here, we propose a novel method to evaluate screw pull-

Corresponding author: Tetsuro Ohba, tooba@yamanashi.ac.jp

Received: April 11, 2018, Accepted: July 4, 2018, Advance Publication: August 25, 2018

Copyright (C) 2019 The Japanese Society for Spine Surgery and Related Research 
out strength clinically using the pull-out length with CTIGN. The purpose of this study was to (1) evaluate the influence of pedicle screw loosening on clinical outcomes; (2) clarify the association between the pull-out length and pedicle screw loosening 1 year after surgery; and (3) determine radiographically which pedicle screw parameters predominantly influence the pull-out strength of PPS.

\section{Methods}

\section{Patient group}

This study was approved by our institutional review board. Written informed consent was received from all eligible patients. This study involved a retrospective analysis of 32 consecutive patients who underwent minimally invasive lumbar or thoracic spinal stabilization using intraoperative CT-IGN without anterior reconstruction by two boardcertified spinal surgeons at a single institute from 2015 to 2016. The inclusion criteria were age $>60$ years and followup for a minimum of 1 year. Basic demographic and surgical data, including age, sex, and bone mineral density (BMD), were collected.

\section{Surgical technique}

The patients were placed in the standard prone position on a Jackson table (Mizuho OSI, Union City, CA). A spinous process bone clamp attached to the reference frame was placed one spinal level above the level of fusion. Navigation tools were registered and the O-arm scanner (Medtronic, Louisville, CO) was then used to obtain intraoperative CT images that were transferred to a computer navigation system (Stealth Medtronic Navigation, Louisville, CO).

Skin incisions were determined based on the pedicle entry point by navigated planned pedicle screw trajectories. A navigated awl was used to enter the center of the bony pedicle canal as determined by the image data viewed in the axial, coronal, and sagittal planes, followed by guide wire placement. Tap and pedicle screws were placed using the guide wire. Before rod connection, further intraoperative CT was used to verify the position of the screws to make sure their placement needed no revision. No revisions were needed in the present treatment group. A titanium alloy rod with a diameter of $5.5 \mathrm{~mm}$ was used for all cases. Rods were inserted from the most cranial skin incisions and set screws were installed from the cranial side to the caudal side.

\section{Radiological evaluation}

All patients underwent postoperative imaging using an eight-slice multi-detector CT system with a $0.83 \mathrm{~mm}$ section thickness (Lightspeed Ultra; GE Healthcare, Milwaukee, WI). Screw positioning was assessed via axial, sagittal, and coronal plane images to determine whether screw breaches occurred medially, laterally, inferiorly, or superiorly. The accuracy of screw placement was evaluated according to crite- ria published by Neo at al. ${ }^{11}$.

The axial and sagittal screw trajectory angles to the midsagittal line and superior endplate at each thoracolumbar vertebral level were measured in the axial and sagittal CT images $^{12)}$ (Fig. 1A, B). After the size of CT images was normalized using the diameter of the same vertebrae, the screw pull-out length was measured in axial CT images scanned both immediately after the pedicle screw was inserted and 17 days postoperatively (Fig. 1C). Screw pull-out was defined as an observation of more than $1 \mathrm{~mm}$ pull-out length. Pedicle screw loosening was defined as a lucent zone around the screw as described previously in axial and sagittal CT images obtained 1 year postoperatively ${ }^{12)}$.

All axial and sagittal trajectory angles, screw pull-out length, and loosening and accuracy of pedicle screw placement were determined by two independent spinal surgeons blinded to the patient data.

\section{Clinical outcomes}

The postoperative baseline patient health status was measured (for lumbar pain-related factors) using the RolandMorris Disability Questionnaire (RDQ), the Oswestry Disability Index (ODI), and the lumbar visual analog scale (LVAS) 1 year after surgery.

\section{Statistical analysis}

All data are reported as means \pm SD. Data were analyzed using two-sided Student $t$ or Fisher exact tests to determine significant differences. All statistical calculations were performed with Prism version 6.0 (Graph Pad Software, La Jolla, CA). $P<0.05$ was considered statistically significant $(* P<0.05, * * P<0.01, * * * P<0.001$, **** $P<$ $0.0001)$.

\section{Results}

Overall, 298 pedicle screws were placed at various levels of the spine as depicted in Fig. 2A. According to NEO grading (for grades 1-3), the overall pedicle breach rate was $2.7 \%(8 / 298)$ of screws. No cases of neurovascular injury were caused by screw placement in either group. As the number of screws that breached a pedicle was small, these screws were excluded from further analysis.

Table 1 summarizes the preoperative baseline characteristics of the patients with (+) or without (-) pedicle screw loosening 1 year after minimally invasive surgery for posterior spinal fusion with intraoperative CT-IGN. No significant differences were observed in the mean age of patients, their sex, average BMD (young adult mean \%), mean stabilized length, or proportion of smokers between groups with (+) and without (-) screw loosening. The ODI, RDQ, and LVAS scores were not significantly different between the groups before surgery $(P>0.05$; Table 2$)$. Two scores, ODI and L-VAS, significantly improved after surgery in all patients in both groups $(P<0.01$; Table 3$)$. These 2 scores at 1 year after surgery were significantly higher in patients in 
A Trajectory angle (Axial)

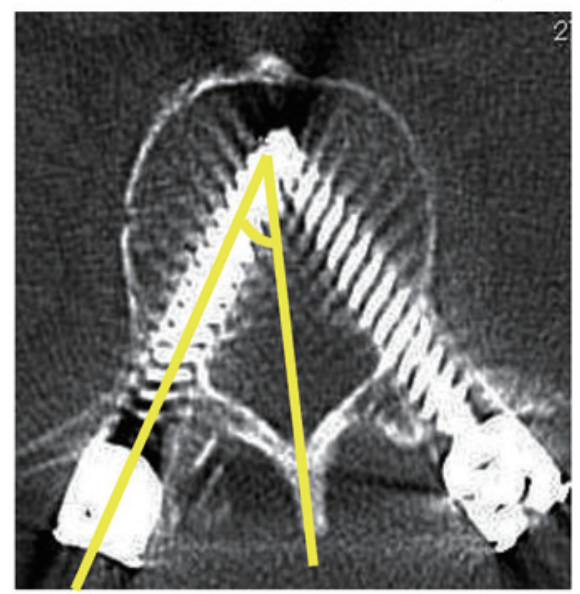

B

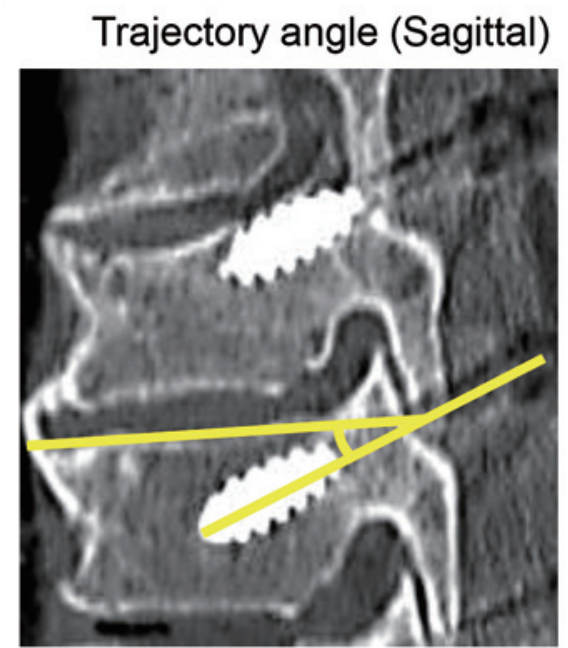

C
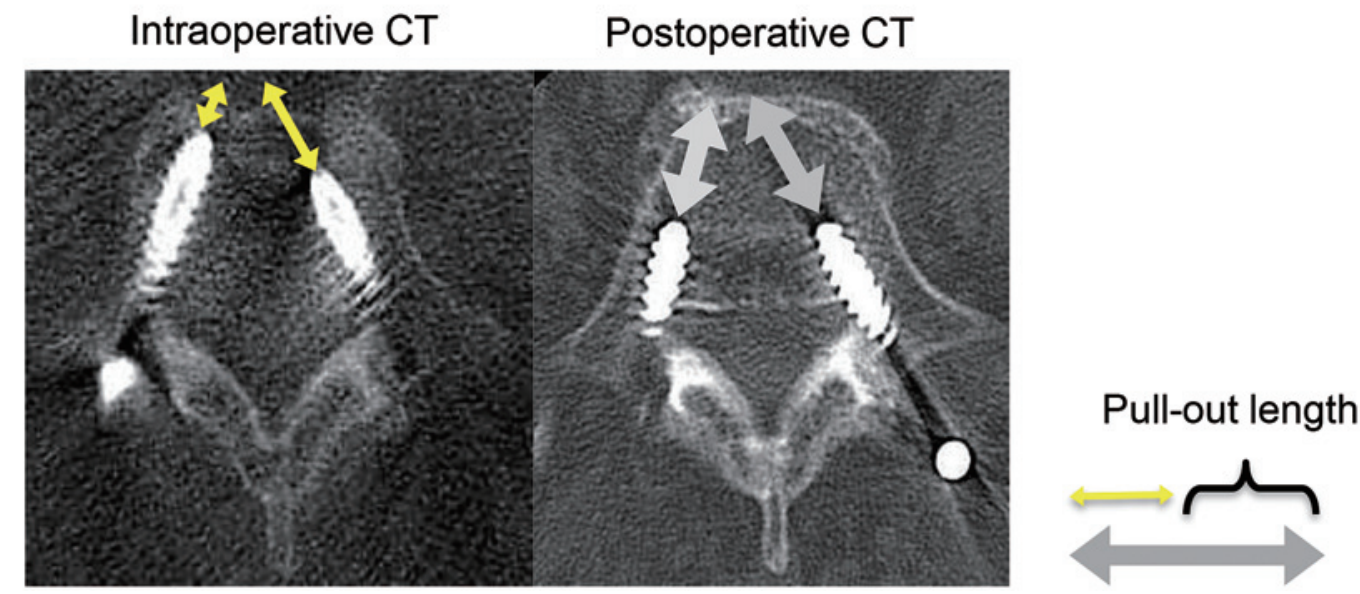

Figure 1. A and B. Graphical definition of axial and sagittal trajectory angles. C. Graphical definition of screw pull-out length. The screw pull-out length was measured in axial CT images scanned both immediately after pedicle screw insertion and postoperatively.

the group with screw loosening $(+)$ than in those in the group without loosening $(-)(P<0.05$; Table 2$)$. By contrast, the RDQ scores 1 year after surgery were not significantly different between the groups $(P>0.05$; Table 2$)$.

The overall pedicle screw pull-out rate was $16.2 \%$ (47/ $290)$ of screws; $8.5 \%$ (4/47) of screw pull-out was observed at the upper instrumented vertebra (UIV) and 59.6\% (28/47) at the lowest instrumented vertebra (LIV) (Fig. 2B). We found multiple pull-out screws in 15 patients, with an average of $2.6 \pm 0.83$ pull-out screws in each case. When we compared the screw pull-out length between the thoracic and lumbar levels, that at the lumbar levels was significantly greater than that at the thoracic levels (supplementary figure).

The overall pedicle screw loosening rate was $15.2 \%$ (44/ $290) ; 11.4 \%$ of screw loosening was observed at the UIV, and $68.2 \%$ was observed at the LIV (Fig. 2C). The diameters, lengths, and trajectory angles (sagittal) of the screws placed were not significantly different between patients in the groups with $(+)$ or without $(-)$ screw loosening (Table
3). By contrast, screws with loosening (+) had significantly lower (axial) trajectories and higher screw pull-out lengths than those without loosening (-) (Table 2). Interestingly, $81.8 \%(36 / 44)$ of patients with loosened pedicle screws had developed the screw pull-out phenomenon postoperatively (Table 3).

\section{Discussion}

Our data showed that the ODI 1 year postoperatively was significantly higher in patients in the group with pedicle screw loosening (+) than in those in the group without screw loosening (-). Additionally, the present study indicated that the screw pull-out phenomenon during rod connection in patients undergoing PPS with intraoperative CTIGN could be evaluated using intra- and postoperative CT. Moreover, screw pull-out was a risk factor for screw loosening, and both screw pull-out and loosening were observed frequently at the LIV. A higher axial trajectory decreased the possibility of screw loosening. 


\section{A}

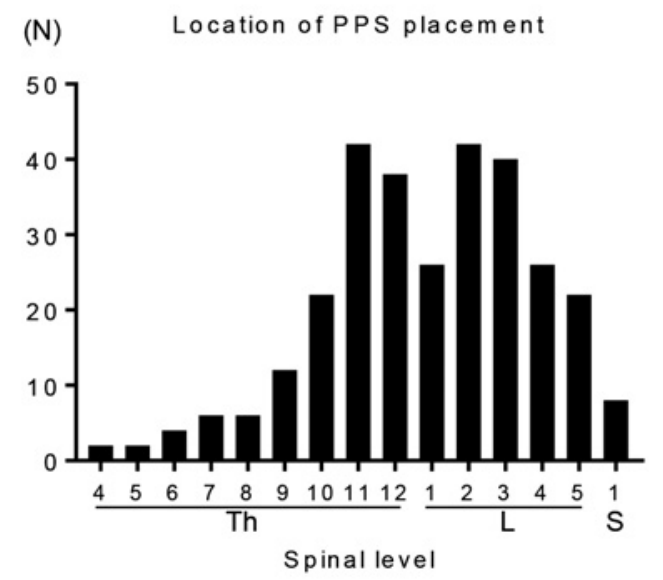

B Location of PS pull-out $(n=47)$

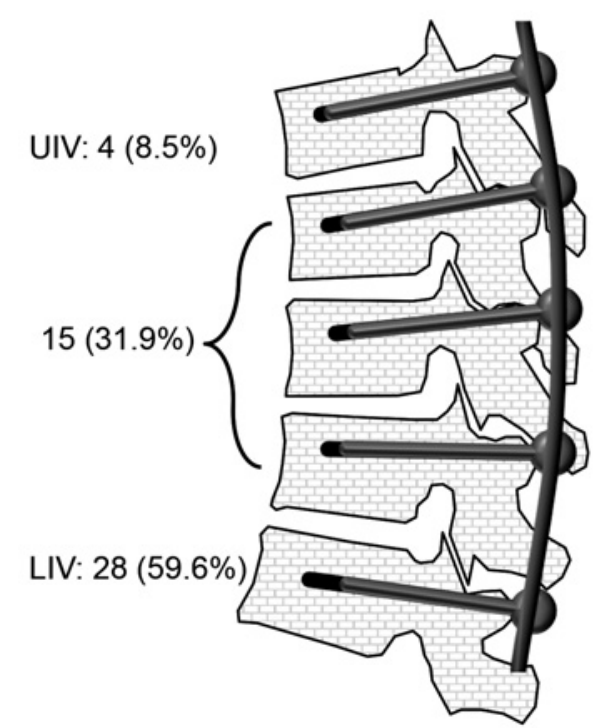

\section{Location of PS loosening $(n=44)$}

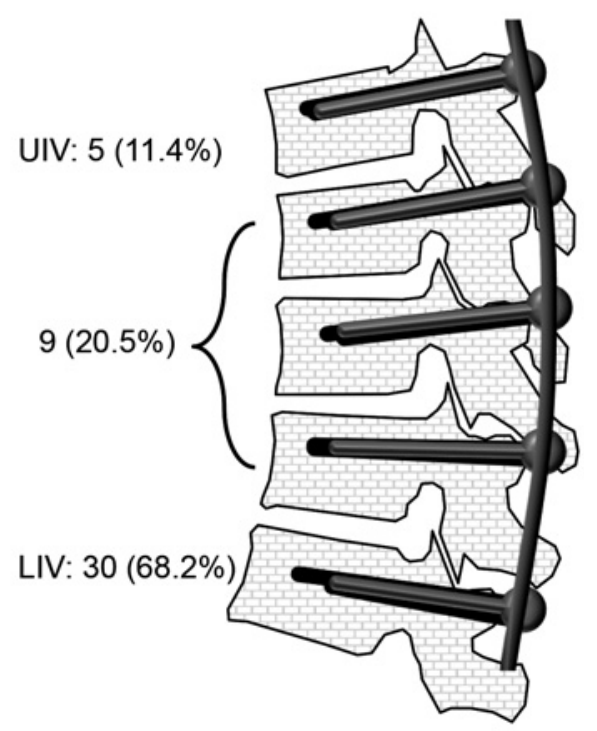

Figure 2. A. Graph showing the spinal level of PPS with CT-IGN. B. Frequency of the screw pull-out phenomenon at the UIV, the LIV, or the interlevel. C. Frequency of screw loosening at the UIV, the LIV, or the interlevel. Abbreviations: PPS=percutaneous pedicle screw; CTIGN=computed tomography image-guided navigation; $\mathrm{N}=$ total number of pedicle screws placed; $\mathrm{S}=$ sacral spine; Th=thoracic spine; $\mathrm{L}=$ lumbar spine; $\mathrm{UIV}=$ upper instrumented vertebra; $\mathrm{LIV}=$ lower instrumented vertebra

Screw loosening is a clinically relevant complication and sometimes causes pain and the need for revision surgery ${ }^{13}$. Our data indicated that screw loosening causes worse ODI and VAS scores 1 year postoperatively. Risk factors for screw loosening have been reported to be older patient age and lower bone density ${ }^{14,15}$. By contrast, clinical evidence of modifiable risk factors for screw loosening was missing ${ }^{14}$. Numerous biomechanical studies in vitro have attempted to clarify crucial factors for the anchorage capacity of pedicle screws, focusing on bone density, insertion depth, misplacement, and insertion angle. However, these studies have inherent limitations associated with a cadaveric study and/or bone models, in which it is difficult to mimic the actual clinical situation, such as the influence of adjacent vertebral musculature and ligaments, and the wide variations in the material properties of bone, living tissues, and postoperative loading ${ }^{4-6,916-22)}$. Therefore, the strengths and clinical relevance of this study are that it showed that a lower axial trajectory and a high screw pull-out force during rod connection might be crucial risk factors for PPS loosening in clinical situations. A rod reduction device has been reported as the cause for screws to be pulled toward the rigid rod, causing a failure of the screw-bone interface in deformity-correction surgery ${ }^{23,24}$. Our study indicated that this pull-out force toward the rod in the PPS system might be a risk factor for screw pull-out during rod connection, especially at the LIV. Therefore, more attention to the screw pull-out force resulting in screw loosening at the LIV in PPS systems might be 
Table 1. Summary of the Preoperative Demographics of Patients with or without PS Loosening 1 Year after Surgery.

\begin{tabular}{lccl}
\hline & \multicolumn{2}{c}{ Loosening } & $P$ \\
\hline Age, *y & $(-)(\mathrm{n}=11)$ & $(+)(\mathrm{n}=21)$ & \\
Sex (female/male) & $64.5 \pm 16.5$ & $72 \pm 8.2$ & 0.098 \\
BMD* (young adult mean \%) & $75.8 \pm 19.5$ & $79 \pm 14$ & 0.9 \\
Stabilized length* & $4.73 \pm 1.0$ & $4.67 \pm 1.28$ & 0.69 \\
Smoker (\%) & $2(25 \%)$ & $3(14.3 \%)$ & 0.99 \\
No. of loosening PS $(\%)$ & $0(0 \%)$ & $44(15.6 \%)$ & \\
Primary diagnosis & & & \\
Trauma & 9 & 16 & \\
Infection & 0 & 2 & \\
Metastasis & 2 & 3 & \\
\hline
\end{tabular}

PS=pedicle screw; BMD=bone mineral density; *mean \pm standard deviation (SD)

Table 2. Comparison of Clinical Outcomes with or without Loosening.

\begin{tabular}{lcccc}
\hline & Overall $(\mathrm{n}=32)$ & $\begin{array}{c}\text { Loosening } \\
(-)(\mathrm{n}=11)\end{array}$ & $(+)(\mathrm{n}=21)$ & $P$ \\
\hline Pain score before surgery & & & & \\
ODI $(\%)$ & $53.9 \pm 27$ & $59 \pm 29$ & $51.3 \pm 26.9$ & 0.59 \\
$\mathrm{RDQ}^{\#}$ (points) & $17 \pm 6.4$ & $14 \pm 8.5$ & $17.9 \pm 5.9$ & 0.38 \\
VAS (Lumbar) & $7.1 \pm 3.7$ & $7.3 \pm 3.2$ & $6.9 \pm 2.6$ & 0.18 \\
Pain score after surgery & & & \\
ODI $^{\#}$ (\%) & $22.8 \pm 19.8$ & $10.8 \pm 9.6$ & $29 \pm 21.9$ & $<0.05^{*}$ \\
RDQ $^{\#}$ (points) & $8.7 \pm 6.8$ & $6 \pm 4.47$ & $10.1 \pm 7.5$ & 0.28 \\
VAS $^{\#}$ (Lumbar) & $3.4 \pm 2.6$ & $1.3 \pm 0.9$ & $4.7 \pm 2.5$ & $<0.05^{*}$ \\
\hline
\end{tabular}

ODI=Oswestry Disability Index; RDQ=Roland-Morris Disability Questionnaire; VAS=visual analog scale

"Mean \pm standard deviation (SD)

$P$ denotes significance of difference between patients in groups with (+) and without (-) loosening. $* P<0.05$

Table 3. Comparison of PS Parameters with or without Loosening.

\begin{tabular}{lccc}
\hline & \multicolumn{2}{c}{ Loosening } & $P$ \\
& $(-)(\mathrm{n}=246)$ & $(+)(\mathrm{n}=44)$ & \\
\hline Screw diameter $^{\#}(\mathrm{~mm})$ & $6.34 \pm 0.87$ & $6.55 \pm 0.87$ & 0.17 \\
Screw length $^{\#}(\mathrm{~mm})$ & $42 \pm 3.47$ & $42.7 \pm 2.94$ & 0.21 \\
${\text { Screw trajectory angle (axial) }{ }^{\#}\left({ }^{\circ}\right)}^{*}$ & $22.3 \pm 9.5$ & $18.9 \pm 9.1$ & $<0.05^{*}$ \\
Screw trajectory angle (sagittal) ${ }^{*}\left({ }^{\circ}\right)$ & $6.63 \pm 5.5$ & $5.18 \pm 3.82$ & 0.098 \\
Screw pull-out length $(\mathrm{mm})$ & $0.114 \pm 0.58$ & $4.31 \pm 4.92$ & $<0.0001^{* * * *}$ \\
No. of screw pull-out $(\%)$ & $11(4.5 \%)$ & $36(81.8 \%)$ & \\
\hline
\end{tabular}

$\mathrm{PS}=$ pedicle screw
${ }^{\#}$ Mean \pm standard deviation $(\mathrm{SD})$
$* P<0.05 ; * * * * P<0.0001$

needed (Fig. 3).

The present study has some limitations, including its comparatively small sample size and that it is not case controlled. CT evaluation after inserting the screw was done during surgery, but CT evaluation after connecting the rod and screw in a reduction maneuver was done after surgery, not during surgery. This study investigated cases without anterior reconstruction, and it is possible that the outcome may change when anterior reconstruction is performed. The study patients were relatively young, and young adult mean $\%$ had an average of $70 \%$ or more, with no bone vulnerability. Further studies focusing on modifiable treatment strategies to avoid screw loosening, including the rod material and diameter; the gap of alignment at the fusion levels and accurate rod bending to decrease the gap of alignment at the fusion levels; the proportion of the pull-out length and the 

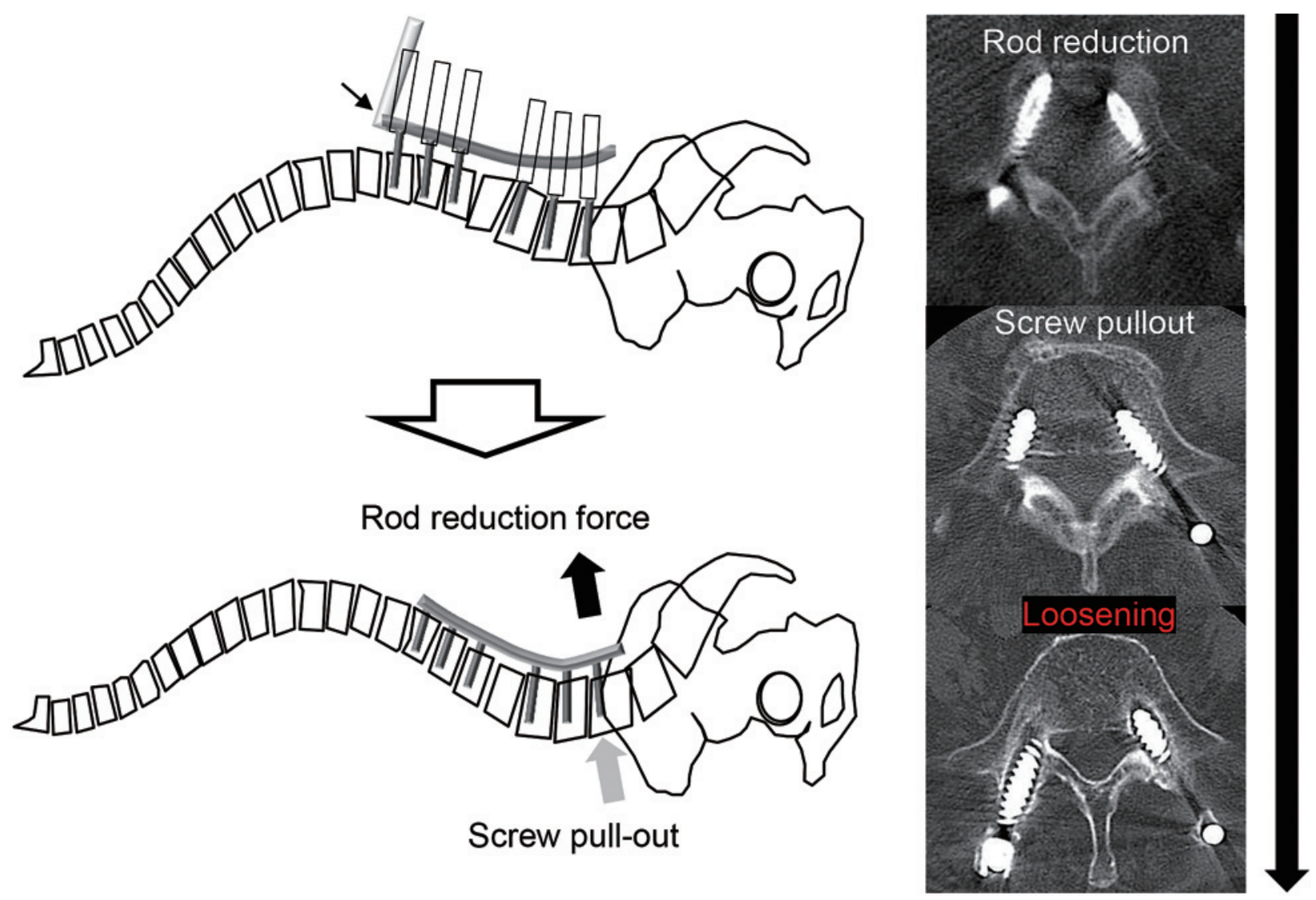

Figure 3. Representative case with screw pull-out during rod reduction, resulting in screw loosening at the LIV. Abbreviations: LIV=lower instrumented vertebra

screw diameter per pedicle width, and the order of the set screws installed are warranted because these factors might affect screw pull-out. Additionally, the results of this study are not satisfactory clinically. Thus far, these patients have only undergone drug treatment, such as with TERIPARATIDE, and need a longer follow-up to determine the outcomes. Hence, the strategy for revision surgery should be established by further studies. Nevertheless, to our knowledge, this is the first study to evaluate the screw pull-out length during rod reduction and to consider associations with PPS loosening.

\section{Conclusions}

A lower axial trajectory and an increased screw pull-out length after rod reduction are crucial risk factors for screw loosening.

Conflicts of Interest: The authors declare that there are no relevant conflicts of interest.

Author Contributions: TO wrote and prepared the manuscript, and all the authors participated in the study design. All authors have read, reviewed, and approved the article.

\section{References}

1. Miller CA, Ledonio CG, Hunt MA, et al. Reliability of the Planned pedicle screw trajectory versus the actual pedicle screw trajectory using intra-operative $3 \mathrm{D} \mathrm{CT}$ and image guidance. Int J Spine Surg. 2016;10:38.

2. Kim MC, Chung HT, Cho JL, et al. Factors affecting the accurate placement of percutaneous pedicle screws during minimally invasive transforaminal lumbar interbody fusion. Eur Spine J. 2011;20 (10):1635-43.

3. La Barbera L, Ottardi C, Villa T. Comparative analysis of international standards for the fatigue testing of posterior spinal fixation systems: the importance of preload in ISO 12189. Spine J. 2015; 15(10):2290-6.

4. Li N, He D, Xing Y, et al. The effect of lateral wall perforation on screw pull-out strength: a cadaveric study. J Orthop Surg Res. 2015;10:6.

5. Moldavsky M, Salloum K, Bucklen B, et al. In vitro biomechanical study of pedicle screw pull-out strength based on different screw path preparation techniques. Indian J Orthop. 2016;50(2): $177-82$.

6. Wichmann JL, Booz C, Wesarg S, et al. Quantitative dual-energy CT for phantomless evaluation of cancellous bone mineral density of the vertebral pedicle: correlation with pedicle screw pull-out strength. Eur Radiol. 2015;25(6):1714-20.

7. Farshad M, Farshad-Amacker NA, Bachmann E, et al. Biomechanical comparison of sagittal-parallel versus non-parallel pedicle screw placement. Acta neurochirurgica. 2014;156(11):2147-51.

8. Inceoglu S, Montgomery WH, Jr., St Clair S, et al. Pedicle screw insertion angle and pullout strength: comparison of 2 proposed strategies. J Neurosurg Spine. 2011;14(5):670-6. 
9. Saraf SK, Singh RP, Singh V, et al. Pullout strength of misplaced pedicle screws in the thoracic and lumbar vertebrae - A cadaveric study. Indian J Orthop. 2013;47(3):238-43.

10. Ohba T, Ebata S, Fujita K, et al. Percutaneous pedicle screw placements: accuracy and rates of cranial facet joint violation using conventional fluoroscopy compared with intraoperative threedimensional computed tomography computer navigation. Eur Spine J. 2016;25(6):1775-80.

11. Neo M, Sakamoto T, Fujibayashi S, et al. The clinical risk of vertebral artery injury from cervical pedicle screws inserted in degenerative vertebrae. Spine. 2005;30(24):2800-5.

12. Ohtori S, Inoue $G$, Orita $S$, et al. Comparison of teriparatide and bisphosphonate treatment to reduce pedicle screw loosening after lumbar spinal fusion surgery in postmenopausal women with osteoporosis from a bone quality perspective. Spine. 2013;38(8):E 487-92.

13. Bredow J, Boese CK, Werner CM, et al. Predictive validity of preoperative CT scans and the risk of pedicle screw loosening in spinal surgery. Arch Orthop Trauma Surg. 2016;136(8):1063-7.

14. Galbusera F, Volkheimer D, Reitmaier S, et al. Pedicle screw loosening: a clinically relevant complication? Eur Spine J. 2015;24(5): 1005-16.

15. Wu ZX, Gong FT, Liu L, et al. A comparative study on screw loosening in osteoporotic lumbar spine fusion between expandable and conventional pedicle screws. Arch Orthop Trauma Surg. 2012; 132(4):471-6.

16. Demir T, Camuscu N, Tureyen K. Design and biomechanical testing of pedicle screw for osteoporotic incidents. Proc Inst Mech Eng H. 2012;226(3):256-62.

17. Cakir E, Eyuboglu EE, Yazar U, et al. May "Dubel" be a solution for pullout problem of the pedicle screws at osteoporotic spine? Turk Neurosurg. 2014;24(5):726-30.

18. Amirouche F, Solitro GF, Magnan BP. Stability and spine pedicle screws fixation strength-a comparative study of bone density and insertion angle. Spine Deform. 2016;4(4):261-7.

19. Schulze M, Gehweiler D, Riesenbeck $O$, et al. Biomechanical characteristics of pedicle screws in osteoporotic vertebraecomparing a new cadaver corpectomy model and pure pull-out testing. J Orthop Res. 2017;35(1):167-74.

20. Varghese V, Saravana Kumar G, Krishnan V. Effect of various factors on pull out strength of pedicle screw in normal and osteoporotic cancellous bone models. Med Eng Phys. 2017;40:28-38.

21. Weiser L, Huber G, Sellenschloh K, et al. Insufficient stability of pedicle screws in osteoporotic vertebrae: biomechanical correlation of bone mineral density and pedicle screw fixation strength. Eur Spine J. 2017;26(11):2891-7.

22. Liebsch $\mathrm{C}$, Zimmermann J, Graf N, et al. In vitro validation of a novel mechanical model for testing the anchorage capacity of pedicle screws using physiological load application. J Mech Behav Biomed Mater. 2018;77:578-85.

23. Abe Y, Ito M, Abumi K, et al. Scoliosis corrective force estimation from the implanted rod deformation using 3D-FEM analysis. Scoliosis. 2015;10(2):S2.

24. Kang DG, Lehman RA, Jr., Wagner SC, et al. Effects of rod reduction on pedicle screw fixation strength in the setting of Ponte osteotomies. Spine J. 2015;15(1):146-52.

Spine Surgery and Related Research is an Open Access journal distributed under the Creative Commons Attribution-NonCommercial-NoDerivatives 4.0 International License. To view the details of this license, please visit (https://creativeco mmons.org/licenses/by-nc-nd/4.0/). 